\title{
Enseignement magistral : Intérêt potentiel de son intégration aux stages hospitaliers et de la réalisation de contrôles de connaissance impromptus
}

\author{
Vincent COTTIN*, Jean-François MORNEX*, Jean-François CORDIER*
}

\begin{abstract}
Résuméc ontexte: Le cours magistral est l'une des modalités essentielles de la formation médicale initiale dans notre université. But : Évaluer l'intérêt potentiel de son intégration aux stages hospitaliers et de la réalisation de contrôles de connaissance impromptus. M éthode : Les mêmes enseignants ont assuré l'enseignement de la pneumologi e simultanément pour deux U nités de F ormation et de Recherche (UFR). Seuls les étudiants de I'U FR1 bénéficiaient d'un enseignement dispensé parallèlement aux stages hospitaliers ( « intégré »), et d'un contrôle impromptu pris en compte dans la note globale. L'assiduité et les résultats à l'examen final ont été comparés entre 33 étudiants de I'UFR1, soit la moitié de l'effectif des étudiants de cette UFR, et 72 étudiants de I'U FR2, soit la totalité de l'effectif de cette UFR, qui ont suivi le même enseignement magistral et la même évaluation certifiante. Résultats : Les étudiants de I'UFR1 étaient significativement plus assidus, ont obtenu plus souvent une note supérieure à la moyenne, et ont obtenu une note moyenne plus élevée que ceux de l'U FR2. Ces différences étaient observées aussi bien pour la note global e que pour chacune des sous épreuves qui la constituent. Conclusion :M al gré l'existence de biais méthodologiques, cette étude et en faveur de l'utilité d'intégrer les cours magi straux et le stage hospitalier, et d'appliquer une mesure d'incitation à l'assiduité et au travail personnel régulier, telle que le contrôle impromptu, en formation médicale initiale.
\end{abstract}

\section{Mots ClésEnseignement ; cours magistral ; stages hospitaliers.}

Summary context: Lectures are one of the essential elements of initial medical formation in our university. Goal: To assess the potential value of its integration to clinical rotations and of impromptu knowledge assesments. Method:T he same lecturers were in charge of teaching lung diseases in two F ormation and Research U nits (FRU) the same year. O nly students from FRU 1 were exposed to lectures given in parallel to hospital instruction courses, and to an unexpected additional examination which was taken into account in the final note. Attendance at lectures and results to the final examination for lung diseases were compared between 33 students from FRU 1, repre senting half of the number of students in this class, and 72 students from FRU 2, the whole class, all had the same teaching and examination. Results: Students from FRU 1 were more often present at the lectures, obtained more often a note above average, and got higher notes than those from FRU 2. Such differences hold true for the final global note as well as for each of the tests.Conclusion:D espite possible methodol ogical biases, our study indicates that integrating lectures and hospital instruction courses, and inciting students to be present at the lectures and to work regularly (by an unexpected examination) may be beneficial in the initial medical formation.

KeywordsTeaching; lectures; hospital instruction courses.

Pédagogie M édicale $2002 ; 3$ : 97-100

\footnotetext{
*Service de Pneumologie - Hôpital Cardiovasculaire et Pneumologique Louis Pradel - Université Caude Bernard Lyon - France.

Correspondance : Vincent Cottin - Service de Pneumologie - Hôpital Cardiovasculare et Pneumologique Louis Pradel 69394 Lyon Cedex 03 - Tel. : (33) 472357269 - Fax : (33) 472357653 -

mailto:vcottin@rockefeller.univ-lyon1.fr
} 


\section{Recherche et Perspectives}

\section{Introduction}

L'enseignement magistral reste la pierre angulaire de la formation médicale initiale actuelle en France'. II présente plusieurs avantages, en permettant idéalement : d'établir un premier contact entre enseignant et étudiant ; d'exposer les objectifs pédagogiques et de donner des éléments de motivation aux étudiants; de délivrer un message structurant accentuant les points essentiels et l'information utile et pertinente, même si l'exposé n'est pas exhaustif ; de présenter une information régulièrement actualisée ; de faciliter la compréhension et la mémorisation par un exposé interactif et illustrépar une iconographie (ce qui est vu et entendu est mieux mémorisé que ce qui est uniquement lu); de conseiller sur les modalités du travail personnel, notamment en ce qui concerne le choix des référentiels; de former les étudiants à la critique scientifique; de répondre aux interrogations des étudiants; etc. A beaucoup d'égards, le cours magistral est d'autant plus contributif qu'il est interactif et permet la communication entre enseignants et étudiants. Cependant, l'enseignement magistral fait l'objet de critiques de la part des étudiants' ; ceux-ci lui reprochent souvent son côté scolaire, passif, consomma teur de temps, et l'accusent parfois d'être peu formateur, avec comme conséquence la faible assiduité des étudiants français à la majorité des cours magistraux, auxquels ils préfèrent d'autres modalités d'apprentissage, tels que les cours polycopiés.

A l'heure où chaque université et chaque enseignant adaptent les modalités d'enseignement aux exigences de la réforme du second cycle des études médicales², il est crucial d'évaluer le bénéfice potentiel des modalités de I'enseignement magistral. D u fait de la difficulté de mener des études randomisées (comparant, par exemple, un enseignement magistral concomitant des stages hospitaliers - «intégré » - ou non), qui compromettraient les principes de l'éthique et de la justice scolaires ${ }^{3}$, nous avons réalisé une étude rétrospective, mettant à profit une particularité locale et transitoire de I'enseignement de la pneumologie dans notre université ; en effet, les mêmes enseignants ont assuré simultanément la formation de cette discipline dans deux Unités de Formation et de Recherche (UFR) distinctes, ce qui permet de comparer l'effet d'un nombre restreint de paramètres qui diffèrent entre ces U FR. Ainsi, nous avons pu évaluer l'intérêt d'un enseignement comportant à la fois l'intégration de l'enseigne- ment magistral et des stages hospitaliers, et une incitation au travail personnel et à l'assiduité sous forme d'un contrôle impromptu, par rapport à une approche plus traditionnelle en France qui comporte un «simple » enseignement magistral.

\section{M éthodes \\ O rganisation générale de l'enseignement}

La population étudiée comporte les étudiants appartenant à deux U FR de I'U niversité Claude B ernard Lyon 1 (que nous appellerons I'UFR1 et I'UFR2). Les modalités d'enseignement étaient extrêmement proches pour les étudiants de ces deux UFR, puisque les cours magistraux et les évaluations (épreuve et correction) étai ent communs (et donc le programme, les objectifs pédagogiques, les méthodes pédagogiques et supports d'enseignement, les enseignants). Q uelques différences pouvaient cependant être relevées entre les deux groupes étudiés. (a) Les étudiants de I'UFR 1 étai ent répartis en deux groupes, et suivaient l'enseignement en deux sessions, en début et en fin d'année universitaire; I'enseignement était en partie « intégré » aux stages hospitaliers. Les étudiants de l'U FR2 ont suivi les cours théoriques en début d'année (avec la première moitié des étudiants de l'UFR1), et pouvaient effectuer un stage hospitalier en pneumologie en cours d'année, non « intégré » aux cours magistraux. (b) Un contrôle impromptu (épreuve rédactionnelle courte réali sée lors d'un cours magistral) était organisé lors de l'enseignement théorique pour les deux U FR, mais sa note n'était prise en compte dans la note globale que pour I'U FR 1, où elle comptait pour $20 \%$ de la note globale. (c) Les étudiants de I'U FR 1 suivai ent majoritairement l'enseignement du certificat de pneumologie en $3^{e}$ année du second cycle des études médicales (32 sur 33, $97 \%$ ) et ceux de l'UFR 2 en $2^{\mathrm{e}}$ année du second cycle (69 sur 75, $92 \%$ ). (d) L'enseignement hospitalier avait lieu dans deux services hospitaliers différents appartenant au centre hospitalo-universitaire. (e) Enfin, le référentiel d'enseignement, lorsqu'il existait, était indiqué lors de chaque cours par l'enseignant, qui conseillait un article de référence ou un chapitre d'ouvrage. Le référentiel conseillé était donc le même dans les deux U FR. C ependant, certains étudiants de I'U FR2 utilisaient également comme référentiel un ancien «polycopié» propreà leur U FR, établi par les enseignants. 


\section{Méthodologie}

II s'agit d'une étude rétrospective, réalisée dans le cadre d'un D iplôme Universitaire de Pédagogie M édicale (Directeur : Pr G . Llorca, Lyon) ${ }^{4}$ Afin de diminuer autant que possible les facteurs confondants pour l'analyse, nous avons choisi de restreindre la comparaison aux deux groupes d'étudiants ayant suivi l'enseignement (et donc l'évaluation) en début d'année universitaire 2001. Cela concerne donc l'ensemble des étudiants de I'U FR2 (72 étudiants), et la moitié de ceux de l'UFR 1 (UFR 1 groupe 1, 33 étudiants) (Figure 1). N ous avons comparé ces deux groupes d'étudiants en ce qui concerne l'assiduité (mesurée par la proportion d'étudiants présents lors du contrôle impromptu des connaissances), et les résultats à l'épreuve de pneumologie de la première session (janvier) en 2001 (pourcentage de réussite à l'examen et note moyenne).

\section{Analyse statistique}

Les variables qualitatives ont été comparées par le test de Chi-2, et les variables quantitatives par une méthode non paramétrique (test $U$ de $M$ ann-W hitney) ${ }^{5}$ à l'aide du logiciel SPSS version 10.0.7 pour W indows.

\section{Figure 1. Représentation graphique des notes globales obtenues à l'épreuve de pneumologie par UFR.} $* p<0,0001$

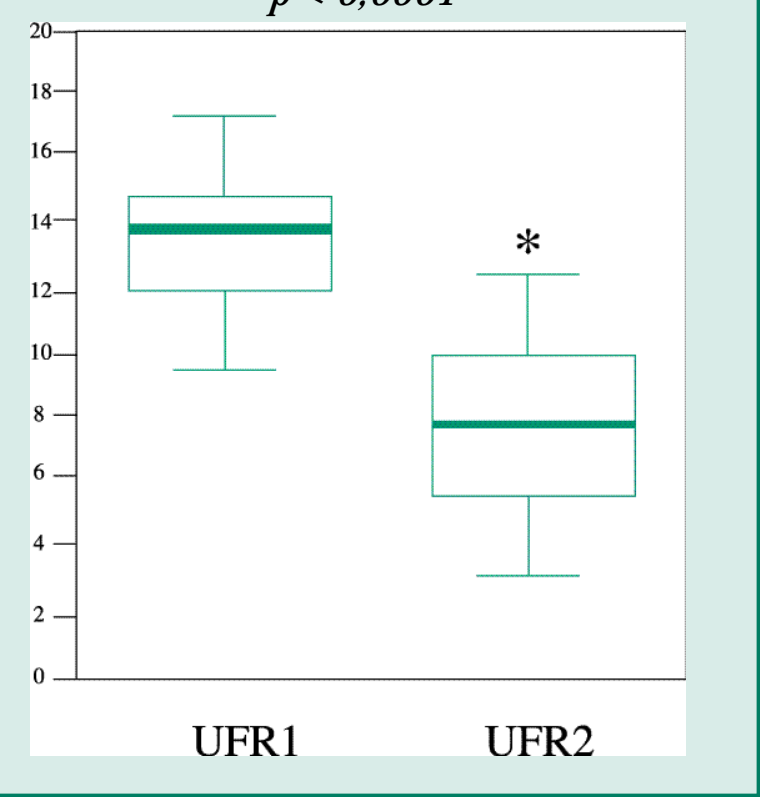

\section{Résultats}

Assiduité

L'assiduitéétait significativement plus importante parmi les étudiants de l'U FR 1 (31 sur 33 présents, soit $91 \%$ ) que parmi ceux de I'UFR2 le jour du contrôle impromptu (13 sur 72 , soit $18 \%, p<0,0001$ ).

\section{Analyse comparative des résultats}

La note globale était en moyenne de $12,02 \pm 0,3$ (SEM) pour les étudiants de l'U FR 1 , contre seulement 7,97 \pm 0,29 pour I'U FR2, soit une différence importante et statistiquement significative ( $p<0,0001$ ) (Figure 1). 27 des 33 étudiants de l'UFR1 (81,8\%) ont obtenu une note globale supérieure à la moyenne, contre seulement 18 des 72 étudiants de l'U FR2 ( $25 \%, p<0,0001)$. De plus, les notes étaient significativement plus élevées pour les étudiants de l'U FR 1 que pour ceux de I'U FR2 pour chacune des sous-épreuves constituant l'épreuve globale (questions à choix multiples, cas cliniques avec questions à choix multiples, cas cliniques avec questions rédactionnelles courtes) (résultats non représentés), indiquant quel'écart de note global e entre les deux U FR ne s'explique pas par une différence isolée portant sur l'une des sous-épreuves, ce qui aurait pu orienter vers un biais attribuable à l'un des enseignants ou l'une des questions.

\section{Discussion}

Notre étude visait à évaluer les modalités de l'enseignement magistral tel qu'il est réalisé dans deux UFR distinctes de notre université pour l'enseignement de la pneumologie. Le principal résultat de ce travail est de démontrer que les étudiants ont mieux réussi les épreuves de l'examen dans I'UFR où (a) les cours magis traux et le stage hospitalier ont été réalisés en parallèle (enseignement dit «intégré ») ; et (b) une mesure d'incitation à l'assiduité au cours magistral et au travail personnel régulier par l'étudiant a été appliquée (le contrôle impromptu pris en compte dans la note finale). M ême si cette étude apporte des éléments en faveur de l'utilité de l'enseignement magistral «intégré », elle ne permet pas de déterminer si l'élément favorable est l'assiduité, ou l'incitation à un travail personnel régulier par le principe du contrôle impromptu, ou enfin l'intégration des stages hospitaliers et des cours magistraux.

L'intégration de l'enseignement magistral et des stages 


\section{Concepts et Innovations}

hospitaliers est pratiqué en pneumologie dans une des deux UFR depuis plus de 15 ans. II joue manifestement un rôle de motivation des étudiants, et favorise l'apprentissage et la mémorisation. II est unanimement apprécié des étudiants, mais son utilité réelle n'avait pas pu encore être appréciée. En revanche, sa mise en œuvre est complexe pour l'université. L'intégration de l'enseignement oblige à la répétition du cycle d'enseignement en cours d'année, multipliant d'autant le nombre annuel d'heures de cours magistral par enseignant. II est surtout difficile de faire coïncider l'ensemble de l'enseignement théorique avec un stage hospitalier dont la durée n'est plus que de trois mois dans notre université. C es résultats doivent, cependant, être interprétés avec prudence, car un certain nombre d'autres facteurs ont pu influencer les résultats : I'année d'étude (les étudiants de l'U FR 1, qui sont plus avancés dans le cursus médical ont plus de connaissances dans les autres disciplines, et ont une plus grande expérience hospitalière, ce qui facilite probablement l'appropriation des connaissances), le lieu de stage, le type de référentiel utilisé, le thème de
I'évaluation (on ne peut exclure que l'évaluation ait porté en partie sur des éléments transmis explicitement par les enseignants lors du cours magistral, mais peu mis en valeur dans les référentiels conseillés aux étudiants), et le fait que seule, la moitié des étudiants de I'U FR 1 ont étéinclus.

\section{En conclusion}

Cette étude préliminaire suggère que la présence au cours magistral, la motivation à un travail personnel, et la réalisation simultanée des stages hospitaliers et de l'enseignement théorique, sont favorables à l'acquisition des connaissances par les étudiants. S'ils sont confirmés, ces résultats sont susceptibles de modifier notre approche de la formation médicale actuelle, dans laquelle la place de l'enseignement magistral et du stage hospitalier est souvent discutée.

Cette information pourrait remettre au goût du jour une modalité d'enseignement souvent critiquée, et constituer un important facteur de motivation, pour les étudiants comme pour les enseignants.

\section{Références}

1.Chabot JM . Les méthodes d'enseignement et d'évalua tion : ce qu'en pensent les étudiants. Rev Prat 1995 ; $45: 1137-8$

2.Cordier JF. Etudes médicales : nouveaux objectifs et programme du 2e cycle. Rev Prat $2000 ; 50$ : 2037-8.

3.Llorca G . La formation médicale (aspects conceptuels). Lyon : Meditions; 1999.
4.Llorca G. La formation à I'enseignement médical à I'université Claude-Bernard - Lyon-1. La lettre du pneumologue $2001 ; 4: 40-1$.

5.M athews DE, Farewell VT. U sing and understanding medical statistics. 3rd ed. Basel (Switzerland): Karger ; 1996 